\title{
Exakt dosiert dank elektrischer Spindelventile
}

D as Unternehmen Axiss erweitert sein

Angebot elektrischer Spindelventile, die das reproduzierbare Dosieren von Kleinstmengen auch bei größeren Kartuschendurchmessern ermöglichen.

Bei den elektrischen Auspresseinheiten V-2K-DUO-E50 bis V-2K-DUOE400 wird der mechanische Hub auf die Kartuschenkolben über eine hochauflösende Spindel ausgeführt, sodass kleinste lineare Bewegungen erreichbar sind.

Die Mischungsverhältnisse sind über die Kartuschengeometrie vorgegeben. Mit dem Einsetzen der jeweiligen Adapter kann das V-2K-DUO-Ventil schnell und einfach auf ein anderes Mischungsverhältnis umgebaut werden. Derzeit sind die Kartuschen mit einem Mischungsverhältnis 1:1, 2:1, 4:1 und 10:1 verfügbar. Für die drei zur Zeit erhältlichen Kartuschengrößen $50 \mathrm{ml}, 200 \mathrm{ml}$ und $400 \mathrm{ml}$ sind entsprechende Ventile erhältlich, mit denen Medien mit einer Viskosität von bis zu 80.000 mpas statisch gemischt und dosiert werden können.
Um reproduzierbare Dosierergebnisse $z u$ erreichen, erfolgt die Eingabe der entsprechenden Parameter wie Dosiergeschwindigkeit, Rückzug, usw. über eine spezielle Steuereinheit mit Folientastatur und LCD-Display. Die Dosiermenge wird über die Spindelsteigung und die Motorschritte eingestellt. Es besteht aber auch die Möglichkeit, die Dosierung unabhängig von der eingestellten Menge direkt über einen externen Signaleingang auszulösen. Dabei kann das Dosiervolumen z. B. über eine externe SPS gesteuert werden.

Der große Vorteil beim Ausdrücken der Komponenten A und B mittels eines Elektromotors mit Spindel ist die von Anfang an gleichbleibende Kraftentwicklung auf den Kolben, die für ein behutsames Anfahren bzw. Ausdrücken des Materials sorgt. Durch die Einstellung der Auspressgeschwindigkeit können bei der Viskosität und dem Abrissverhalten am Ende der Dosierung spürbare Verbesserungen erzielt werden.

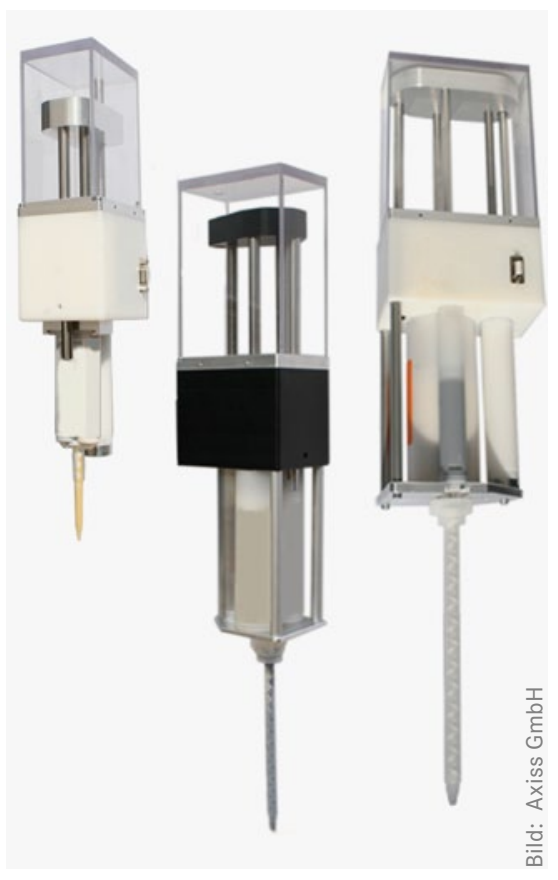

Auch durch die Einstellung eines Rückzuges der Kolben zur Entlastung des Materials nach dem Dosiervorgang wird ein wesentlich besserer Materialstopp nach dem Dosiervorgang erreicht.

Weitere Infos: Axiss GmbH, 75210 Keltern, www.axiss.de

\section{Prüfstand zur Messung von Torsionsschwingungen}

— ür die Analyse des Schwingverhaltens

- von Rotationssystemen hat das Fraunhofer LBF einen Prüfstand entwickelt, mit dem rotatorisch wirksame Systeme sowie passive, adaptive und aktive Tilger sowie Schwingungsdämpfer vermessen werden können. Durch den Einsatz elektrodynamischer Schwingerreger ist eine hohe Prüffrequenz möglich. Es können damit auch motornahe Komponenten mit sehr großen Schwingungsamplituden vermessen werden. Der Prüfstand lässt sich an die jeweilige Aufgabenstellung anpassen und erlaubt somit einen flexiblen Einsatz. Die Neuentwicklung eines Prüfstands ist bei Bedarf ebenfalls möglich.

Die Langzeitmessung rotatorisch höherfrequent schwingender Komponenten gestattet die Untersuchung des Betriebsverhaltens. Ergänzend zur Prüfung können komplexe Schwingformen mittels Laservibrometrie oder Bildkorrelation grafisch dargestellt und verschiedene Sensordaten wie Kraft, Beschleunigung, Weg und Temperatur aufgenommen werden.

Der neue Prüfstand wurde bereits bei verschiedenen Untersuchungen eingesetzt - so zum Beispiel im Automobilbereich für Langzeituntersuchungen sowohl des Betriebsverhaltens als auch des Schadensauftritts rotatorisch höherfrequent schwingender Komponenten.

Mit seiner Hilfe wurde zudem ein Rotationsschwingungsneutralisator entwickelt, dessen Betriebspunkt an eine sich ändernde Anregungsfrequenz angepasst

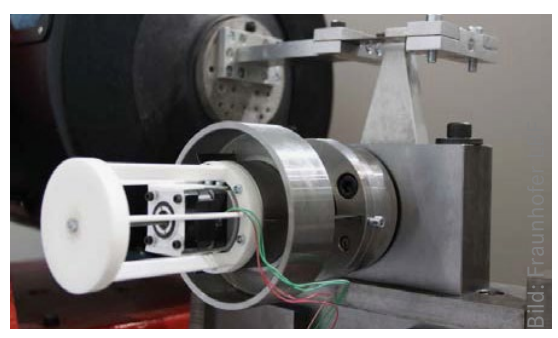

Rotationsschwingungsprüfstand mit adaptivem Tilger

werden kann. Die Anpassung der Eigenfrequenz geschieht dabei durch die Änderung der aktiven Länge von entsprechenden elastischen Elementen.

Weitere Infos: Fraunhofer-Institut für Betriebsfestigkeit und Systemzuverlässigkeit LBF, www.lbf.fraunhofer.de 